\title{
On Self-Adaptive Method for General Mixed Variational Inequalities
}

\author{
Abdellah Bnouhachem, ${ }^{1,2}$ Muhammad Aslam Noor, ${ }^{3}$ and Eman H. Al-Shemas ${ }^{4}$ \\ ${ }^{1}$ School of Management Science and Engineering, Nanjing University, Nanjing 210093, China \\ ${ }^{2}$ Ibn Zohr University, Présidence, BP 32/S, 80000 Agadir, Morocco \\ ${ }^{3}$ Mathematics Department, Faculty of Sciences, COMSATS Institute of Information Technology, \\ Plot no. 30, Sector H-8, Islamabad, Pakistan \\ ${ }^{4}$ Mathematics Department, College of Basic Education, Main Campus, Shamiyah, Adiliya 73251, Kuwait
}

Correspondence should be addressed to Muhammad Aslam Noor, noormaslam@hotmail.com

Received 13 September 2007; Accepted 17 April 2008

Recommended by Alois Steindl

\begin{abstract}
We suggest and analyze a new self-adaptive method for solving general mixed variational inequalities, which can be viewed as an improvement of the method of (Noor 2003). Global convergence of the new method is proved under the same assumptions as Noor's method. Some preliminary computational results are given to illustrate the efficiency of the proposed method. Since the general mixed variational inequalities include general variational inequalities, quasivariational inequalities, and nonlinear (implicit) complementarity problems as special cases, results proved in this paper continue to hold for these problems.
\end{abstract}

Copyright $(2008$ Abdellah Bnouhachem et al. This is an open access article distributed under the Creative Commons Attribution License, which permits unrestricted use, distribution, and reproduction in any medium, provided the original work is properly cited.

\section{Introduction}

Variational inequalities introduced in the early sixties have played a critical and significant part in the study of several unrelated problems arising in finance, economics, network analysis, transportation, elasticity, and optimization. Variational inequalities theory has witnessed an explosive growth in theoretical advances, algorithmic development, and applications across all disciplines of pure and applied sciences, see [1-16]. A useful and important generalization of variational inequalities is the mixed variational inequality containing a nonlinear term $\varphi$. But the applicability of the projection method is limited due to the fact that it is not easy to find the projection except in very special cases. Secondly, the projection method cannot be applied to suggest iterative algorithms for solving general mixed variational inequalities involving the nonlinear term $\varphi$. This fact has motivated many authors to develop the auxiliary principle technique for solving the mixed variational inequalities. In recent years, several techniques have been developed to suggest and analyze various iterative methods for solving 
different types of variational inequalities. It is worth mentioning that if the nonlinear term in the variational inequalities is a proper, convex, and semilower continuous function, then it is well known that the variational inequalities involving the nonlinear term $\varphi$ are equivalent to the fixed point problems and the resolvent equations. In [11], Noor solved the general mixed variational inequality problem by using the resolvent equations technique. Inspired and motivated by the results of Noor [11], we propose a new method for solving general mixed variational inequalities by using a new direction with a new step size $\alpha_{k}$. We prove the global convergence of the proposed method under the same assumptions as in [11]. An example is given to illustrate the efficiency and its comparison with the results of Noor $[11,14]$. This shows that the method is robust and efficient. This new method can be viewed as an important and significant improvement of Noor and other methods.

\section{Preliminaries}

Let $H$ be a real Hilbert space, whose inner product and norm are denoted by $\langle\cdot, \cdot\rangle$ and $\|\cdot\|$, let $I$ be the identity mapping on $H$, and $T, g: H \rightarrow H$ be two operators. Let $\partial \varphi$ denotes the subdifferential of function $\varphi$, where $\varphi: H \rightarrow R \cup\{+\infty\}$ is a proper convex lower semicontinuous function on $H$. It is well known that the subdifferential $\partial \varphi$ is a maximal monotone operator. We consider the problem of finding $u^{*} \in H$ such that

$$
\left\langle T\left(u^{*}\right), g(v)-g\left(u^{*}\right)\right\rangle+\varphi(g(v))-\varphi\left(g\left(u^{*}\right)\right) \geq 0, \quad \forall g(v) \in H,
$$

which is known as the mixed general variational inequality, see Noor [11]. We also note that the general variational inequality can be written in the equivalent form as find $u^{*} \in H$ such that

$$
0 \in T u+\partial \varphi(g(u)),
$$

which is known as the problem of finding a zero of sum of two(more) monotone operators. It is well known that a wide class of linear and nonlinear problems arising in pure and applied sciences can be studied via the general mixed variational inequalities, see [1-16] and the references therein.

If $K$ is a closed convex set in $H$ and $\varphi(v) \equiv I_{K}(v), \forall v \in H$, where

$$
I_{K}(v)= \begin{cases}0 & \text { if } v \in K, \\ +\infty, & \text { otherwise }\end{cases}
$$

is the indicator function of $K$, then the problem (2.1) is equivalent to finding $u^{*} \in H$ such that $g\left(u^{*}\right) \in K$ and

$$
\left\langle T\left(u^{*}\right), g(v)-g\left(u^{*}\right)\right\rangle \geq 0, \quad \forall g(v) \in K .
$$

Problem (2.4) is called the general variational inequality, which was first introduced and studied by Noor [9] in 1988. For the applications, formulation, and numerical methods of general variational inequalities (2.4), we refer the reader to the survey, see $[1-3,7,12,13,16]$.

If $g \equiv I$, then the problem (2.4) is equivalent to finding $u^{*} \in K$ such that

$$
\left\langle T\left(u^{*}\right), v-u^{*}\right\rangle \geq 0, \quad \forall v \in K,
$$

which is known as the classical variational inequality introduced and studied by Stampacchia [17]. 
Lemma 2.1 (see [4]). For a given $w \in H, z \in H$, and $\rho>0$, the inequality

$$
\langle w-z, z-v\rangle+\rho \varphi(v)-\rho \varphi(z) \geq 0, \quad \forall v \in H
$$

holds if and only if $z=J_{\varphi}(w)$, where $J_{\varphi}=(I+\rho \partial \varphi)^{-1}$ is the resolvent operator.

It follows from Lemma 2.1 that

$$
\left\langle w-J_{\varphi}(w), J_{\varphi}(w)-v\right\rangle+\rho \varphi(v)-\rho \varphi\left(J_{\varphi}(w)\right) \geq 0, \quad \forall v, w \in H .
$$

If $\varphi$ is the indicator function of a closed convex set $K$ in $H$, then the resolvent operator $J_{\varphi}(\cdot)=P_{K}$, where $P_{K}$ is the projection of $H$ onto the closed convex set $K$. It is well known that $J_{\varphi}$ is nonexpansive, that is,

$$
\left\|J_{\varphi}(u)-J_{\varphi}(v)\right\| \leq\|u-v\|, \quad \forall u, v \in H .
$$

Lemma 2.2 (see [10]). $u^{*}$ is solution of problem (2.1) if and only if $u^{*} \in H$ satisfies the relation:

$$
g\left(u^{*}\right)=J_{\varphi}\left[g\left(u^{*}\right)-\rho T\left(u^{*}\right)\right]
$$

where $J_{\varphi}=(I+\rho \partial \varphi)^{-1}$ is the resolvent operator .

From Lemma 2.2, it is clear that $u^{*}$ is solution of (2.1) if and only if $u^{*}$ is a zero point of the function

$$
r\left(u^{*}, \rho\right):=g\left(u^{*}\right)-J_{\varphi}\left[g\left(u^{*}\right)-\rho T\left(u^{*}\right)\right] .
$$

In [11], Noor used the fixed-point formulation (2.9) and the resolvent equations to suggest and analyze the following algorithm for solving problem (2.1).

Algorithm 2.3. For a given $u^{0} \in H$, compute the approximate solution $u^{k+1}$ by the iterative schemes.

Predictor step

$$
g\left(w^{k}\right)=J_{\varphi}\left[g\left(u^{k}\right)-\rho_{k} T\left(u^{k}\right)\right]
$$

where $\rho_{k}$ satisfies

$$
\rho_{k}\left\langle T\left(u^{k}\right)-T\left(g^{-1}\left(J_{\varphi}\left[g\left(u^{k}\right)-\rho_{k} T\left(u^{k}\right)\right]\right)\right), r\left(u^{k}, \rho_{k}\right)\right\rangle \leq \delta\left\|r\left(u^{k}, \rho_{k}\right)\right\|^{2}, \quad \delta \in(0,1) .
$$

Corrector step

$$
g\left(u^{k+1}\right)=J_{\varphi}\left[g\left(u^{k}\right)-\alpha_{k} d\left(u^{k}, \rho_{k}\right)\right]
$$

where

$$
\begin{gathered}
d\left(u^{k}, \rho_{k}\right)=r\left(u^{k}, \rho_{k}\right)-\rho_{k} T\left(u^{k}\right)+\rho_{k} T\left(g^{-1}\left(J_{\varphi}\left[g\left(u^{k}\right)-\rho_{k} T\left(u^{k}\right)\right]\right)\right), \\
\alpha_{k}=\frac{(1-\delta)\left\|r\left(u^{k}, \rho_{k}\right)\right\|^{2}}{\left\|d\left(u^{k}, \rho_{k}\right)\right\|^{2}}
\end{gathered}
$$

is the corrector step size.

If $\varphi$ is an indicator function of a closed convex set $K$ in $H$, then $J_{\varphi} \equiv P_{K}$ [10], the projection of $H$ onto $K$ and consequently Algorithm 2.3 collapses. 
Algorithm 2.4 (see [11]). For a given $u^{0} \in H$, compute the approximate solution $u^{k+1}$ by the iterative schemes.

Predictor step

$$
g\left(w^{k}\right)=P_{K}\left[g\left(u^{k}\right)-\rho_{k} T\left(u^{k}\right)\right]
$$

where $\rho_{k}$ satisfies

$$
\rho_{k}\left\langle T\left(u^{k}\right)-T\left(g^{-1}\left(P_{K}\left[g\left(u^{k}\right)-\rho_{k} T\left(u^{k}\right)\right]\right)\right), r\left(u^{k}, \rho_{k}\right)\right\rangle \leq \delta\left\|r\left(u^{k}, \rho_{k}\right)\right\|^{2}, \quad \delta \in(0,1) .
$$

Corrector step

$$
g\left(u^{k+1}\right)=P_{K}\left[g\left(u^{k}\right)-\alpha_{k} d\left(u^{k}, \rho_{k}\right)\right]
$$

where

$$
\begin{gathered}
d\left(u^{k}, \rho_{k}\right)=r\left(u^{k}, \rho_{k}\right)-\rho_{k} T\left(u^{k}\right)+\rho_{k} T\left(g^{-1}\left(P_{K}\left[g\left(u^{k}\right)-\rho_{k} T\left(u^{k}\right)\right]\right)\right), \\
\alpha_{k}=\frac{(1-\delta)\left\|r\left(u^{k}, \rho_{k}\right)\right\|^{2}}{\left\|d\left(u^{k}, \rho_{k}\right)\right\|^{2}}
\end{gathered}
$$

is the corrector step size.

Throughout this paper, we make following assumptions.

Assumptions

(i) $H$ is finite dimension space.

(ii) $g$ is homeomorphism on $H$, that is, $g$ is bijective, continuous and $g^{-1}$ is continuous.

(iii) $T$ is continuous and g-pseudomonotone operator on $H$, that is,

$$
\left\langle T(u), g\left(u^{\prime}\right)-g(u)\right\rangle \geq 0 \Longrightarrow\left\langle T\left(u^{\prime}\right), g\left(u^{\prime}\right)-g(u)\right\rangle \geq 0 \quad \forall u^{\prime}, u \in H .
$$

(iv) The solution set of problem (2.1) denoted by $S^{*}$ is nonempty.

\section{Iterative method and basic results}

In this section, we suggest and analyze a new method for solving mixed general variational inequality (2.1) by using a new direction with a new step size $\alpha_{k}$, and this is the main motivation of this paper.

\section{Algorithm 3.1}

Step 1. Given $\gamma \in[1,2), \rho_{0}>0, \delta \in(0,1), u^{0} \in H$, and $k=0$.

Step 2. Set

$$
w^{k}=g^{-1}\left(J_{\varphi}\left[g\left(u^{k}\right)-\rho_{k} T\left(u^{k}\right)\right]\right) .
$$


Step 3. If

$$
r_{k}:=\frac{\left\|\rho_{k}\left(T\left(u^{k}\right)-T\left(w^{k}\right)\right)\right\|}{\left\|g\left(u^{k}\right)-g\left(w^{k}\right)\right\|} \leq \delta,
$$

then set

$$
\begin{gathered}
\varepsilon^{k}=\rho_{k}\left(T\left(w^{k}\right)-T\left(u^{k}\right)\right), \\
d_{1}\left(u^{k}, \rho_{k}\right):=g\left(u^{k}\right)-g\left(w^{k}\right)+\varepsilon^{k}, \\
d\left(u^{k}, \rho_{k}\right):=g\left(u^{k}\right)-g\left(w^{k}\right)+\rho_{k} T\left(w^{k}\right), \\
\phi\left(u^{k}, \rho_{k}\right):=\left\langle g\left(u^{k}\right)-g\left(w^{k}\right), d_{1}\left(u^{k}, \rho_{k}\right)\right\rangle
\end{gathered}
$$

the stepsize

$$
\alpha_{k}:=\frac{\phi\left(u^{k}, \rho_{k}\right)}{\left\|d\left(u^{k}, \rho_{k}\right)\right\|^{2}}
$$

and the next iterate

$$
\begin{aligned}
g\left(u^{k+1}\right) & =J_{\varphi}\left[g\left(u^{k}\right)-\gamma \alpha_{k} d\left(u^{k}, \rho_{k}\right)\right], \\
\rho_{k} & := \begin{cases}1.5 * \rho_{k} & \text { if } r_{k} \leq 0.5, \\
\rho_{k} & \text { otherwise }\end{cases}
\end{aligned}
$$

$\rho_{k+1}=\rho_{k}$ and $k:=k+1$; go to Step 2 .

Step 4 . Reduce the value of $\rho_{k}$ by $\rho_{k}:=2 / 3 * \rho_{k} * \min \left\{1,1 / r_{k}\right\}$;

set $w^{k}=g^{-1}\left(J_{\varphi}\left[g\left(u^{k}\right)-\rho_{k} T\left(u^{k}\right)\right]\right)$ and go to Step 3 .

If $\varphi$ is an indicator function of a closed convex set $K$ in $H$, then $J_{\varphi} \equiv P_{K}$ [10], the projection of $H$ onto K. Consequently, Algorithm 3.1 reduces to Algorithm 3.2 for solving the general variational inequalities (2.4).

\section{Algorithm 3.2}

Step 1. Given $\gamma \in[1,2), \rho_{0}>0, \delta \in(0,1), u^{0} \in H$, and $k=0$.

Step 2. Set

$$
w^{k}=g^{-1}\left(P_{K}\left[g\left(u^{k}\right)-\rho_{k} T\left(u^{k}\right)\right]\right)
$$

Step 3. If

$$
r_{k}:=\frac{\left\|\rho_{k}\left(T\left(u^{k}\right)-T\left(w^{k}\right)\right)\right\|}{\left\|g\left(u^{k}\right)-g\left(w^{k}\right)\right\|} \leq \delta,
$$


then set

$$
\begin{gathered}
\varepsilon^{k}:=\rho_{k}\left(T\left(w^{k}\right)-T\left(u^{k}\right)\right), \\
d_{1}\left(u^{k}, \rho_{k}\right):=g\left(u^{k}\right)-g\left(w^{k}\right)+\varepsilon^{k}, \\
d\left(u^{k}, \rho_{k}\right):=g\left(u^{k}\right)-g\left(w^{k}\right)+\rho_{k} T\left(w^{k}\right), \\
\phi\left(u^{k}, \rho_{k}\right):=\left\langle g\left(u^{k}\right)-g\left(w^{k}\right), d_{1}\left(u^{k}, \rho_{k}\right)\right\rangle
\end{gathered}
$$

the stepsize

$$
\alpha_{k}:=\frac{\phi\left(u^{k}, \rho_{k}\right)}{\left\|d\left(u^{k}, \rho_{k}\right)\right\|^{2}}
$$

and the next iterate

$$
\begin{aligned}
g\left(u^{k+1}\right)= & P_{K}\left[g\left(u^{k}\right)-\gamma \alpha_{k} d\left(u^{k}, \rho_{k}\right)\right], \\
\rho_{k} & := \begin{cases}1.5 * \rho_{k} & \text { if } r_{k} \leq 0.5, \\
\rho_{k} & \text { otherwise, }\end{cases}
\end{aligned}
$$

$\rho_{k+1}=\rho_{k}$ and $k:=k+1$; go to Step 2 .

Step 4 . Reduce the value of $\rho_{k}$ by $\rho_{k}:=2 / 3 * \rho_{k} * \min \left\{1,1 / r_{k}\right\}$;

set $w^{k}=g^{-1}\left(P_{K}\left[g\left(u^{k}\right)-\rho_{k} T\left(u^{k}\right)\right]\right)$ and go to Step 3 .

Remark 3.3. Equation (3.2) implies that

$$
\left|\left\langle g\left(u^{k}\right)-g\left(w^{k}\right), \varepsilon^{k}\right\rangle\right| \leq \delta\left\|g\left(u^{k}\right)-g\left(w^{k}\right)\right\|^{2}, \quad 0<\delta<1 .
$$

The next lemma shows that $\alpha_{k}$ and $\phi\left(u^{k}, \rho_{k}\right)$ are lower bounded away from zero, whenever $u^{k} \neq w^{k}$.

Lemma 3.4. For given $u^{k} \in H$ and $\rho_{k}>0$, let $w^{k}$ and $\varepsilon^{k}$ satisfy to (3.1) and (3.3), then

$$
\begin{gathered}
\phi\left(u^{k}, \rho_{k}\right) \geq(1-\delta)\left\|g\left(u^{k}\right)-g\left(w^{k}\right)\right\|^{2}, \\
\alpha_{k} \geq \frac{(1-\delta)\left\|g\left(u^{k}\right)-g\left(w^{k}\right)\right\|^{2}}{\left\|d\left(u^{k}, \rho_{k}\right)\right\|^{2}} .
\end{gathered}
$$

Proof. It follows from (3.4) and (3.14) that

$$
\begin{aligned}
\phi\left(u^{k}, \rho_{k}\right) & :=\left\langle g\left(u^{k}\right)-g\left(w^{k}\right), d_{1}\left(u^{k}, \rho_{k}\right)\right\rangle \\
& =\left\|g\left(u^{k}\right)-g\left(w^{k}\right)\right\|^{2}+\left\langle g\left(u^{k}\right)-g\left(w^{k}\right), \varepsilon^{k}\right\rangle \\
& \geq(1-\delta)\left\|g\left(u^{k}\right)-g\left(w^{k}\right)\right\|^{2} .
\end{aligned}
$$

Otherwise, we have

$$
\alpha_{k}:=\frac{\left\langle g\left(u^{k}\right)-g\left(w^{k}\right), d_{1}\left(u^{k}, \rho_{k}\right)\right\rangle}{\left\|d\left(u^{k}, \rho_{k}\right)\right\|^{2}} \geq \frac{(1-\delta)\left\|g\left(u^{k}\right)-g\left(w^{k}\right)\right\|^{2}}{\left\|d\left(u^{k}, \rho_{k}\right)\right\|^{2}},
$$

we can get the assertion of this lemma. 
The next lemma shows that $\|r(u, \rho)\|$ is a nondecreasing function with respect to $\rho$, which can be proved using the techniques as in [1].

Lemma 3.5. For all $u \in H$ and $\rho^{\prime}>\rho>0$, it holds that

$$
\left\|r\left(u, \rho^{\prime}\right)\right\| \geq\|r(u, \rho)\| .
$$

Next lemma has already been studied in [11].

Lemma 3.6. For all $u \in H, u^{*} \in S^{*}$, and $\rho>0$, we have

$$
\left\langle g\left(u^{k}\right)-g\left(u^{*}\right), d\left(u^{k}, \rho_{k}\right)\right\rangle \geq \phi\left(u^{k}, \rho_{k}\right),
$$

where $d\left(u^{k}, \rho_{k}\right)$ and $\phi\left(u^{k}, \rho_{k}\right)$ are defined in (3.5) and (3.6), respectively.

\section{Convergence analysis}

In this section, we prove the global convergence of the proposed method. The following result plays a crucial role in the convergence analysis of the proposed method.

Theorem 4.1. Let $u^{*} \in H$ be a solution of problem (2.1) and let $u^{k+1}$ be the sequence obtained from Algorithm 3.1. Then $u^{k}$ is bounded and

$$
\left\|g\left(u^{k+1}\right)-g\left(u^{*}\right)\right\|^{2} \leq\left\|g\left(u^{k}\right)-g\left(u^{*}\right)\right\|^{2}-\gamma(2-\gamma)(1-\delta)^{2} \frac{\left\|g\left(u^{k}\right)-g\left(w^{k}\right)\right\|^{4}}{\left\|d\left(u^{k}, \rho_{k}\right)\right\|^{2}}
$$

Proof. Let $u^{*} \in H$ be a solution of problem (2.1). Then

$$
\begin{aligned}
\left\|g\left(u^{k+1}\right)-g\left(u^{*}\right)\right\|^{2} & \leq\left\|g\left(u^{k}\right)-g\left(u^{*}\right)-\gamma \alpha_{k} d\left(u^{k}, \rho_{k}\right)\right\|^{2} \\
& =\left\|g\left(u^{k}\right)-g\left(u^{*}\right)\right\|^{2}-2 \gamma \alpha_{k}\left\langle g\left(u^{k}\right)-g\left(u^{*}\right), d\left(u^{k}, \rho_{k}\right)\right\rangle+\gamma^{2} \alpha_{k}^{2}\left\|d\left(u^{k}, \rho_{k}\right)\right\|^{2} \\
& \leq\left\|g\left(u^{k}\right)-g\left(u^{*}\right)\right\|^{2}-2 \gamma \alpha_{k} \phi\left(u^{k}, \rho_{k}\right)+\gamma^{2} \alpha_{k} \phi\left(u^{k}, \rho_{k}\right) \\
& \leq\left\|g\left(u^{k}\right)-g\left(u^{*}\right)\right\|^{2}-\gamma(2-\gamma)(1-\delta)^{2} \frac{\left\|g\left(u^{k}\right)-g\left(w^{k}\right)\right\|^{4}}{\left\|d\left(u^{k}, \rho_{k}\right)\right\|^{2}},
\end{aligned}
$$

where the first inequality follows from the nonexpansiveness of the resolvent operator, the second inequality follows from (3.7) and (3.19), and the third inequality follows from (3.15). Since $\gamma \in[1,2)$ and $\delta \in(0,1)$, we have

$$
\left\|g\left(u^{k+1}\right)-g\left(u^{*}\right)\right\| \leq\left\|g\left(u^{k}\right)-g\left(u^{*}\right)\right\| \leq \cdots \leq\left\|g\left(u^{0}\right)-g\left(u^{*}\right)\right\| .
$$

Since $g$ is homeomorphism, it is easy to verify that the sequence $u^{k}$ is bounded.

We now prove the convergence of Algorithm 3.1.

Theorem 4.2. The sequence $\left\{u^{k}\right\}$ generated by the Algorithm 3.1 converges to a solution of problem (2.1). 
Proof. It follows from (4.1) that

$$
\sum_{k=0}^{\infty} \frac{\left\|g\left(u^{k}\right)-g\left(w^{k}\right)\right\|^{4}}{\left\|d\left(u^{k}, \rho_{k}\right)\right\|^{2}}<\infty
$$

which means that

$$
\lim _{k \rightarrow \infty}\left\|g\left(u^{k}\right)-g\left(w^{k}\right)\right\|=0 .
$$

Since $g$ is homeomorphism, we have

$$
\lim _{k \rightarrow \infty}\left\|u^{k}-w^{k}\right\|=0
$$

This implies that $\left\{w^{k}\right\}$ is bounded. Since $\left\|r\left(u^{k}, \rho\right)\right\|$ is a nondecreasing function of $\rho$, it follows from $\rho_{k} \geq \rho_{\min }$ that

$$
\begin{aligned}
\left\|r\left(w^{k}, \rho_{\min }\right)\right\| & \leq\left\|r\left(w^{k}, \rho_{k}\right)\right\| \\
& =\left\|g\left(w^{k}\right)-J_{\varphi}\left[g\left(w^{k}\right)-\rho_{k} T\left(w^{k}\right)\right]\right\| \\
(\operatorname{using}(3.1) \text { and (3.3)) } & =\left\|J_{\varphi}\left[g\left(u^{k}\right)-\rho_{k} T\left(w^{k}\right)+\varepsilon^{k}\right]-J_{\varphi}\left[g\left(w^{k}\right)-\rho_{k} T\left(w^{k}\right)\right]\right\| \\
& \leq\left\|g\left(u^{k}\right)-g\left(w^{k}\right)+\varepsilon^{k}\right\| \\
(\operatorname{using}(3.2)) & \leq(1+\delta)\left\|g\left(u^{k}\right)-g\left(w^{k}\right)\right\|
\end{aligned}
$$

and from (4.5), we get

$$
\lim _{k \rightarrow \infty} r\left(w^{k}, \rho_{\min }\right)=0
$$

Let $\bar{u}$ be a cluster point of $\left\{w^{k}\right\}$ and the subsequence $\left\{w^{k_{j}}\right\}$ converges to $\bar{u}$. Since $r(u, \rho)$ is a continuous function of $u$, it follows from (4.8) that

$$
r\left(\bar{u}, \rho_{\min }\right)=\lim _{j \rightarrow \infty} r\left(w^{k_{j}}, \rho_{\min }\right)=0 .
$$

From Lemma 2.2, it follows that $\bar{u}$ is a solution point of problem (2.1). Note that inequality (4.1) is true for all solution point of problem (2.1), hence we have

$$
\left\|g\left(u^{k+1}\right)-g(\bar{u})\right\| \leq\left\|g\left(u^{k}\right)-g(\bar{u})\right\|, \quad \forall k \geq 0 .
$$

Since $\left\{g\left(w^{k_{j}}\right)\right\} \rightarrow g(\bar{u})$ and $g\left(u^{k}\right)-g\left(w^{k}\right) \rightarrow 0$, for any given $\varepsilon>0$, there is an $l>0$, such that

$$
\left\|g\left(w^{k_{l}}\right)-g(\bar{u})\right\|<\frac{\varepsilon}{2}, \quad\left\|g\left(u^{k_{l}}\right)-g\left(w^{k_{l}}\right)\right\|<\frac{\varepsilon}{2} .
$$

Therefore, for any $k \geq k_{l}$, it follows from (4.10) and (4.11) that

$$
\left\|g\left(u^{k}\right)-g(\bar{u})\right\| \leq\left\|g\left(u^{k_{l}}\right)-g(\bar{u})\right\| \leq\left\|g\left(u^{k_{l}}\right)-g\left(w^{k_{l}}\right)\right\|+\left\|g\left(w^{k_{l}}\right)-g(\bar{u})\right\|<\varepsilon
$$


and thus the sequence $\left\{g\left(u^{k}\right)\right\}$ converges to $g(\bar{u})$. Using $g$ is homeomorphism, we see that the sequence $\left\{u^{k}\right\}$ converges to $\bar{u}$.

We now prove that the sequence $\left\{u^{k}\right\}$ has exactly one cluster point. Assume that $\tilde{u}$ is another cluster point and satisfies

$$
\delta:=\|g(\tilde{u})-g(\bar{u})\|>0 .
$$

Since $\bar{u}$ is a cluster point of the sequence $\left\{u^{k}\right\}$ and $g$ is homeomorphism, there is a $k_{0}>0$ such that

$$
\left\|g\left(u^{k_{0}}\right)-g(\bar{u})\right\| \leq \frac{\delta}{2} .
$$

On the other hand, since $\bar{u} \in S^{*}$ and from (4.1), we have

$$
\left\|g\left(u^{k}\right)-g(\bar{u})\right\| \leq\left\|g\left(u^{k_{0}}\right)-g(\bar{u})\right\| \quad \forall k \geq k_{0}
$$

it follows that

$$
\left\|g\left(u^{k}\right)-g(\widetilde{u})\right\| \geq\|g(\widetilde{u})-g(\bar{u})\|-\left\|g\left(u^{k}\right)-g(\bar{u})\right\| \geq \frac{\delta}{2} \quad \forall k \geq k_{0} .
$$

This contradicts the assumption that $\tilde{u}$ is cluster point of $\left\{u^{k}\right\}$. Thus the sequence $\left\{u^{k}\right\}$ converges to $\bar{u} \in S^{*}$.

\section{Numerical results}

In this section, we present some numerical results for the proposed method. In order to verify the theoretical assertions, we consider the following problems:

$$
\begin{array}{ll}
\min & h(u)=\sum_{j=1}^{n} u_{j} \log \left(\frac{u_{j}}{p_{j}}\right), \\
\text { s.t. } & A u \in \Pi, \\
& u \geq 0,
\end{array}
$$

where $A$ is an $n \times n$ matrix, $\Pi$ is a simple closed convex set in $R^{n}, 0<p \in R^{n}$ is a parameter vector. Here, the statement that the set $\Pi$ is simple means that the projection onto $\Pi$ is simple to carry out. For the same reason given in Fletcher (see [5, page 222]), each element of the optimal solution of problem (5.1) is positive. Thus the bounds $u \geq 0$ are inactive and can be ignored, therefore problem (5.1) can be written as

$$
\begin{array}{ll}
\min & h(u)=\sum_{j=1}^{n} u_{j} \log \left(\frac{u_{j}}{p_{j}}\right), \\
\text { s.t. } & A u-v=0 \\
& v \in \Pi .
\end{array}
$$

By attaching the Lagrange multiplier $y \in R^{n}$ to the equality constraints $A u-v=0$, the Lagrange function of problem (5.2) is

$$
L(u, v, y)=h(u)-y^{T}(A u-v)
$$


which is defined on $R^{n} \times \Pi \times R^{n}$. If $\left(u^{*}, v^{*}, y^{*}\right) \in R^{n} \times \Pi \times R^{n}$ is a KKT point of problem (5.2), then we have

$$
\begin{aligned}
\nabla h\left(u^{*}\right)-A^{T} y^{*} & =0, \\
\left(v-v^{*}\right)^{T} y^{*} & \geq 0, \quad \forall v \in \Pi, \\
A u^{*}-v^{*} & =0 .
\end{aligned}
$$

Note that problem (5.1) is invariant under multiplication $h$ by some positive scalar $\rho$. Denoting $f(u)=\nabla h(u)$ and eliminating $v$ and $v^{*}$ in (5.4), we see that problem (5.1) is equivalent to a general variational inequality problem. Find $w^{*}$ such that

$$
Q\left(w^{*}\right) \in \Omega, \quad\left[Q\left(v_{1}\right)-Q\left(w^{*}\right)\right]^{T} F\left(w^{*}\right) \geq 0, \quad \forall Q\left(v_{1}\right) \in \Omega,
$$

where

$$
w=\left(\begin{array}{l}
u \\
y
\end{array}\right), \quad Q(w)=\left(\begin{array}{c}
u \\
A u
\end{array}\right), \quad F(w)=\left(\begin{array}{c}
\rho f(u)-A^{T} y \\
y
\end{array}\right), \quad \Omega=R^{n} \times \Pi .
$$

It is well known (see [7, Theorem 1]) that solving (5.5)-(5.6) is equivalent to finding a zero point of the function

$$
e(w):=Q(w)-P_{\Omega}[Q(w)-F(w)]=\left(\begin{array}{c}
\rho f(u)-A^{T} y \\
A u-P_{\Pi}[A u-y]
\end{array}\right) .
$$

Then, solving (5.5)-(5.6) is equivalent to find a pair $\left(u^{*}, y^{*}\right)$, such that

$$
\begin{gathered}
\rho f\left(u^{*}\right)=A^{T} y^{*}, \\
g\left(u^{*}\right) \in \Pi, \quad\left(g(v)-g\left(u^{*}\right)\right)^{T} y^{*} \geq 0, \quad \forall g(v) \in \Pi,
\end{gathered}
$$

where

$$
g(u)=A u
$$

In this case Algorithms 2.3 and 3.1 collapse to Algorithms 2.4 and 3.2, respectively.

In the test, we let $v^{\prime} \in R^{n}$ be a randomly generated vector, $v_{j}^{\prime} \in(-0.5,0.5)$, and $A=$ $I-2\left(v^{\prime} v^{\prime T}\right) /\left(v^{\prime T} v^{\prime}\right)$ be an $n \times n$ Householder matrix. Let

$$
u_{j}^{*} \in(0.1,1.1), \quad y_{i}^{*} \in(-0.5,0.5) .
$$

Note that

$$
f_{j}\left(u^{*}\right)=\left(\nabla h\left(u^{*}\right)\right)_{j}=\log \left(u_{j}^{*}\right)-\log \left(p_{j}\right)+1 .
$$

Since

$$
f\left(u^{*}\right)=A^{T} y^{*},
$$


Table 1: Numerical results for problem (5.9) with $n=200$.

\begin{tabular}{lcccccc}
\hline$\rho$ & \multicolumn{3}{c}{ Algorithm 3.2 } & \multicolumn{3}{c}{ Algorithm 2.4 } \\
No. It. & CPU(Sec.) & $\left\|u^{k}-u^{*}\right\|$ & No. It. & CPU(Sec.) & $\left\|u^{k}-u^{*}\right\|$ \\
\hline $10^{5}$ & 37 & 2.17 & $1.4 * 10^{-15}$ & 307 & 4.31 & $1.12 * 10^{-7}$ \\
$10^{4}$ & 32 & 1.80 & $1.4 * 10^{-15}$ & 377 & 4.33 & $1.16 * 10^{-7}$ \\
$10^{2}$ & 20 & 1.05 & $1.4 * 10^{-15}$ & 293 & 2.81 & $1.22 * 10^{-7}$ \\
1 & 9 & 0.46 & $1.4 * 10^{-15}$ & 321 & 2.66 & $1.22 * 10^{-7}$ \\
$10^{-1}$ & 3 & 0.75 & $1.4 * 10^{-15}$ & 280 & 2.25 & $1.21 * 10^{-7}$ \\
$10^{-3}$ & 6 & 1.62 & $1.4 * 10^{-15}$ & 11484 & 51.23 & $9.54 * 10^{-8}$ \\
\hline
\end{tabular}

Table 2: Numerical results for problem (5.9) with $n=300$.

\begin{tabular}{lcccccc}
\hline$\rho$ & \multicolumn{3}{c}{ Algorithm 3.2} & \multicolumn{3}{c}{ Algorithm 2.4 } \\
No. It. & CPU(Sec.) & $\left\|u^{k}-u^{*}\right\|$ & No. It. & CPU(Sec.) & $\left\|u^{k}-u^{*}\right\|$ \\
\hline $10^{5}$ & 39 & 2.47 & $1.88 * 10^{-15}$ & 539 & 8.39 & $9.36 * 10^{-8}$ \\
$10^{4}$ & 34 & 1.95 & $1.88 * 10^{-15}$ & 645 & 9.53 & $9.71 * 10^{-8}$ \\
$10^{2}$ & 22 & 1.37 & $1.88 * 10^{-15}$ & 527 & 6.81 & $9.5 * 10^{-8}$ \\
1 & 11 & 0.66 & $1.88 * 10^{-15}$ & 572 & 6.43 & $9.45 * 10^{-8}$ \\
$10^{-1}$ & 5 & 0.84 & $1.88 * 10^{-15}$ & 516 & 6.21 & $9.18 * 10^{-8}$ \\
$10^{-3}$ & 5 & 1.75 & $1.88 * 10^{-15}$ & 11532 & 120.01 & $9.56 * 10^{-8}$ \\
\hline
\end{tabular}

we set

$$
\begin{aligned}
& p_{j}=u_{j}^{*} \exp \left(1-e_{j}^{T} A^{T} y^{*}\right), \\
& \Pi=\left\{z \mid l_{B} \leq z \leq u_{B}\right\}
\end{aligned}
$$

where

$$
\begin{aligned}
& \left(l_{B}\right)_{i}= \begin{cases}\left(A u^{*}\right)_{i} & \text { if } y_{i}^{*} \geq 0 \\
\left(A u^{*}\right)_{i}+y_{i}^{*} & \text { otherwise }\end{cases} \\
& \left(u_{B}\right)_{i}= \begin{cases}\left(A u^{*}\right)_{i} & \text { if } y_{i}^{*}<0 \\
\left(A u^{*}\right)_{i}+y_{i}^{*} & \text { otherwise }\end{cases}
\end{aligned}
$$

In this way, we have

$$
A u^{*} \in \Pi, \quad A u^{*}=P_{\Pi}\left[A u^{*}-y^{*}\right]
$$

In all the tests, we take $\delta=0.95$ and $\gamma=1.95$. The calculations are started with a vector $u^{0}$, whose elements are randomly chosen in $(0,1)$ and stopped, whenever $\|r(u, \rho)\|_{\infty} \leq 10^{-7}$.

Since $u^{*}$ is known, we also report the distance $\left\|u^{k}-u^{*}\right\|$ after $\|r(u, \rho)\|_{\infty} \leq 10^{-7}$. All codes are written in Matlab and run on a P4-2.00G note book computer. We test the problem with dimensions $n=200$ and $n=300$. The iteration numbers and the computational time for Algorithms 2.4 and 3.2 with different dimensions and initial parameter $\rho$ are given in the Tables 1-2, and for Algorithm 3.2 and the method of Noor [14] in Tables 3-4. 
Table 3: Numerical results for problem (5.9) with $n=200$.

\begin{tabular}{lcccccc}
\hline$\rho$ & \multicolumn{3}{c}{ Algorithm 3.2 } & \multicolumn{3}{c}{ The method in [14] } \\
& No. It. & CPU(Sec.) & $\left\|u^{k}-u^{*}\right\|$ & No. It. & CPU(Sec.) & $\left\|u^{k}-u^{*}\right\|$ \\
\hline $10^{4}$ & 32 & 1.80 & $1.4 * 10^{-15}$ & 42 & 3.17 & $1.4 * 10^{-15}$ \\
$10^{2}$ & 20 & 1.05 & $1.4 * 10^{-15}$ & 32 & 2.56 & $1.4 * 10^{-15}$ \\
10 & 15 & 1.51 & $1.4 * 10^{-15}$ & 25 & 1.56 & $1.4 * 10^{-15}$ \\
$10^{-1}$ & 3 & 0.75 & $1.4 * 10^{-15}$ & 15 & 1.15 & $1.4 * 10^{-15}$ \\
$10^{-5}$ & 16 & 2.56 & $1.4 * 10^{-15}$ & 715 & 7.78 & $9.49 * 10^{-8}$ \\
$10^{-6}$ & 22 & 2.86 & $1.4 * 10^{-15}$ & 6651 & 44.99 & $9.18 * 10^{-8}$ \\
\hline
\end{tabular}

Table 4: Numerical results for problem (5.9) with $n=300$.

\begin{tabular}{lcccccc}
\hline$\rho$ & \multicolumn{3}{c}{ Algorithm 3.2} & \multicolumn{3}{c}{ The method in [14] } \\
& No. It. & CPU(Sec.) & $\left\|u^{k}-u^{*}\right\|$ & No. It. & CPU(Sec.) & $\left\|u^{k}-u^{*}\right\|$ \\
\hline $10^{4}$ & 34 & 1.95 & $1.88 * 10^{-15}$ & 46 & 3.45 & $1.88 * 10^{-15}$ \\
$10^{2}$ & 22 & 1.37 & $1.88 * 10^{-15}$ & 36 & 2.16 & $1.88 * 10^{-15}$ \\
10 & 17 & 1.56 & $1.88 * 10^{-15}$ & 29 & 1.75 & $1.88 * 10^{-15}$ \\
$10^{-1}$ & 5 & 0.84 & $1.88 * 10^{-15}$ & 16 & 1.79 & $1.88 * 10^{-15}$ \\
$10^{-5}$ & 15 & 2.78 & $1.88 * 10^{-15}$ & 408 & 10.51 & $1.88 * 10^{-15}$ \\
$10^{-6}$ & 21 & 2.31 & $1.88 * 10^{-15}$ & 4057 & 82.82 & $1.88 * 10^{-15}$ \\
\hline
\end{tabular}

From Tables 1-4, we could see that Algorithms 2.4 and the method in [14] work well, if $\rho$ is sufficient large. If the parameter $\rho$ is too small, then the iteration numbers and the computational time can increase significantly. Also these tables show that Algorithm 3.2 is very efficient for the problem tested. In addition, for our method, it seems that the computational time and the iteration numbers are not very sensitive to the problem size.

\section{Acknowledgments}

The authors would like to thank the referees and Professor Dr. Alois Steindl for their very constructive comments and suggestions. Abdellah Bnouhachem was supported by china Postdoctoral science Foundation Grant no. 20060390915 and the National Key Technology R\&D Program (2006BAH02A06).

\section{References}

[1] A. Bnouhachem, "A self-adaptive method for solving general mixed variational inequalities," Journal of Mathematical Analysis and Applications, vol. 309, no. 1, pp. 136-150, 2005.

[2] A. Bnouhachem, "A new step size rule in Noor's method for solving general variational inequalities," The Australian Journal of Mathematical Analysis and Applications, vol. 4, no. 1, article 12, 10 pages, 2007.

[3] A. Bnouhachem and M. A. Noor, "Numerical comparison between prediction-correction methods for general variational inequalities," Applied Mathematics and Computation, vol. 186, no. 1, pp. 496-505, 2007.

[4] H. Brezis, Operateurs Maximaux Monotone et Semigroupes de Contractions dans les Espace d'Hilbert, NorthHolland, Amsterdam, The Netherlands, 1973.

[5] R. Fletcher, Practical Methods of Optimization, A Wiley-Interscience Publication, John Wiley \& Sons, Chichester, UK, 2nd edition, 1987.

[6] R. Glowinski, J.-L. Lions, and R. Trémolières, Numerical Analysis of Variational Inequalities, vol. 8 of Studies in Mathematics and Its Applications, North-Holland, Amsterdam, The Netherlands, 1981. 
[7] B. He, "Inexact implicit methods for monotone general variational inequalities," Mathematical Programming, vol. 86, no. 1, pp. 199-217, 1999.

[8] J.-L. Lions and G. Stampacchia, "Variational inequalities," Communications on Pure and Applied Mathematics, vol. 20, no. 3, pp. 493-519, 1967.

[9] M. A. Noor, "General variational inequalities," Applied Mathematics Letters, vol. 1, no. 2, pp. 119-122, 1988.

[10] M. A. Noor, "An implicit method for mixed variational inequalities," Applied Mathematics Letters, vol. 11, no. 4, pp. 109-113, 1998.

[11] M. A. Noor, "Pseudomonotone general mixed variational inequalities," Applied Mathematics and Computation, vol. 141, no. 2-3, pp. 529-540, 2003.

[12] M. A. Noor, "New extragradient-type methods for general variational inequalities," Journal of Mathematical Analysis and Applications, vol. 277, no. 2, pp. 379-394, 2003.

[13] M. A. Noor, "Some developments in general variational inequalities," Applied Mathematics and Computation, vol. 152, no. 1, pp. 199-277, 2004.

[14] M. A. Noor, "On general mixed quasi variational inequalities," Journal of Optimization Theory and Applications, vol. 120, no. 3, pp. 579-599, 2003.

[15] M. A. Noor, "Fundamentals of mixed quasi variational inequalities," International Journal of Pure and Applied Mathematics, vol. 15, no. 2, pp. 137-258, 2004.

[16] M. A. Noor and A. Bnouhachem, "On an iterative algorithm for general variational inequalities," Applied Mathematics and Computation, vol. 185, no. 1, pp. 155-168, 2007.

[17] G. Stampacchia, "Formes bilinéaires coercitives sur les ensembles convexes," Comptes Rendus de L'Académie des Sciences, vol. 258, pp. 4413-4416, 1964. 


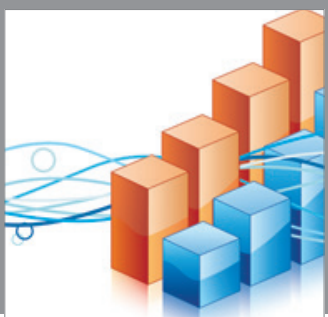

Advances in

Operations Research

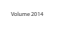

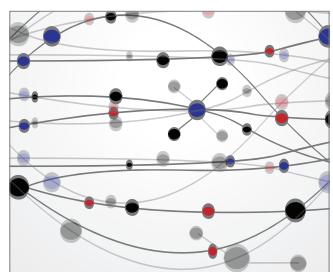

\section{The Scientific} World Journal
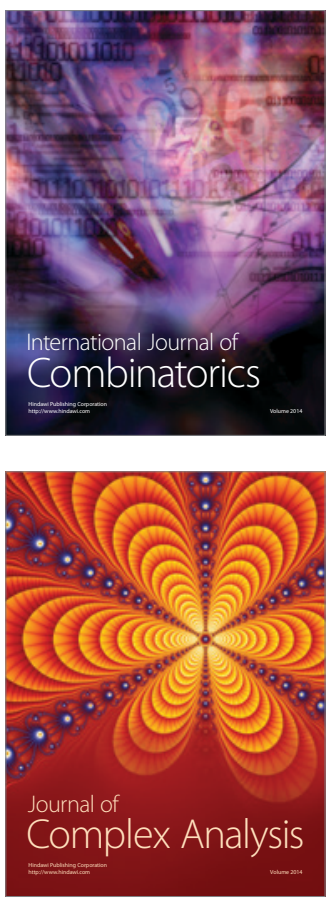

International Journal of

Mathematics and

Mathematical

Sciences
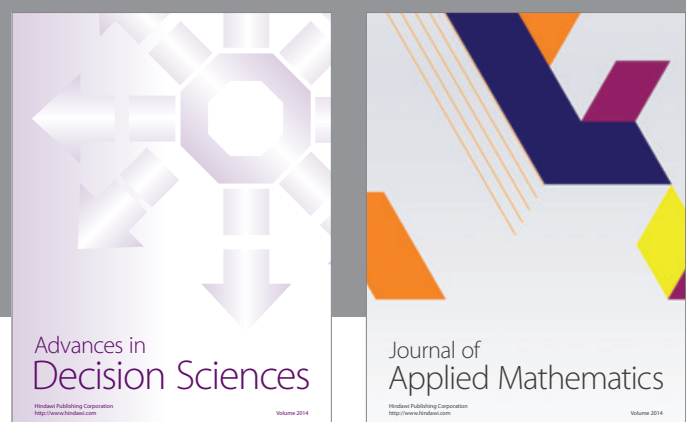

Journal of

Applied Mathematics
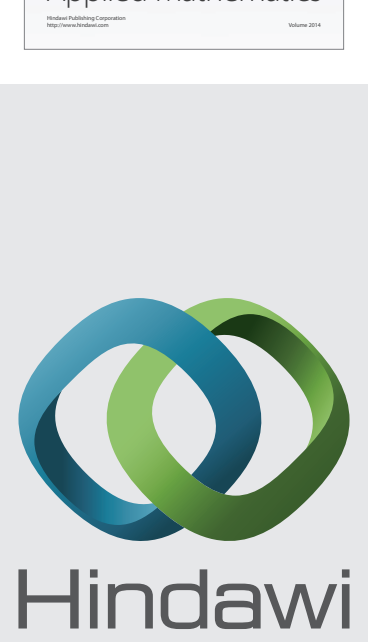

Submit your manuscripts at http://www.hindawi.com
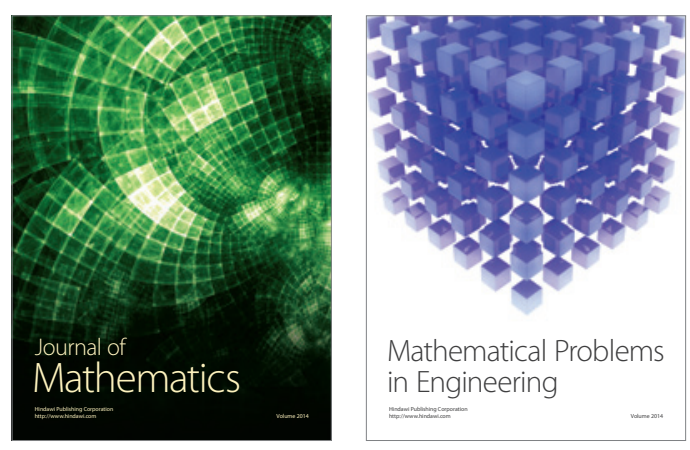

Mathematical Problems in Engineering
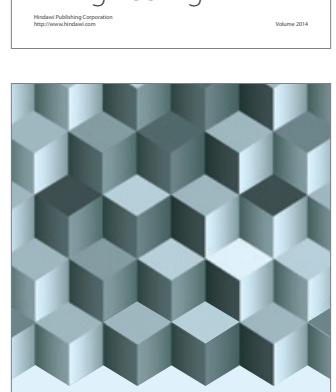

Journal of

Function Spaces
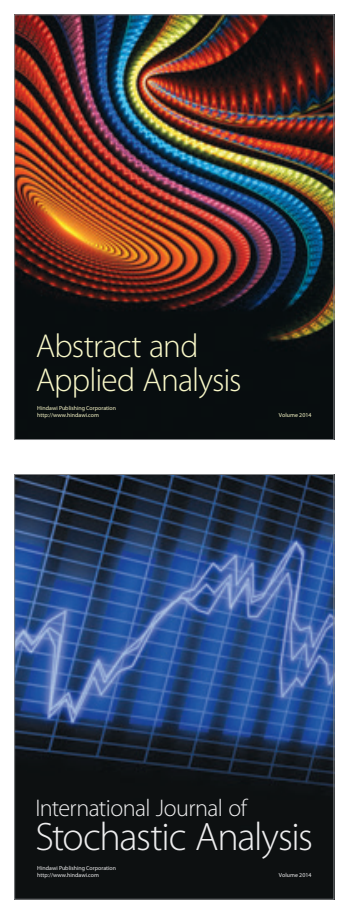

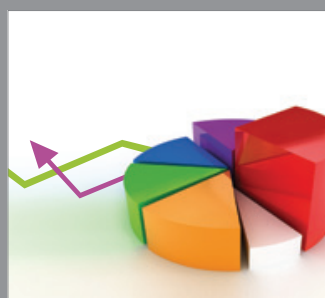

ournal of

Probability and Statistics

Promensencen
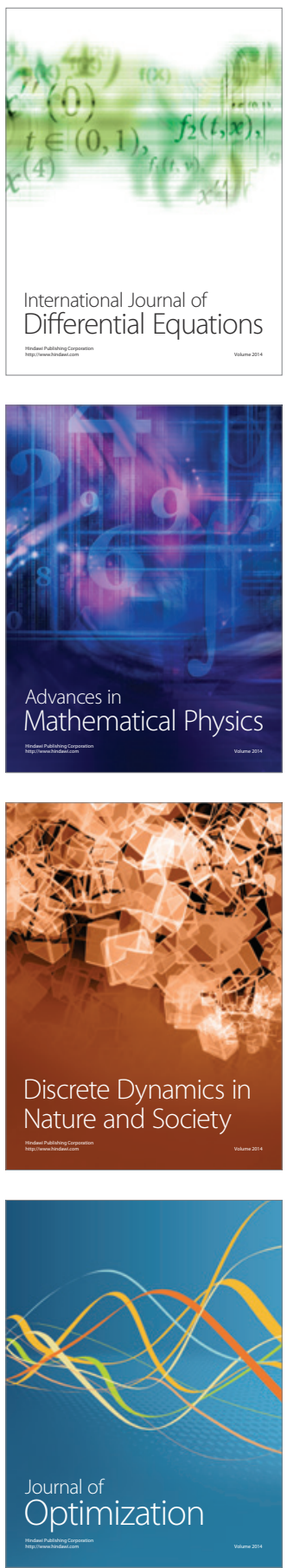